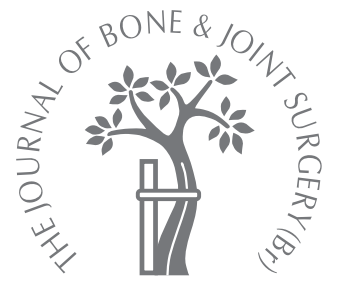

C. Maynou,

X. Cassagnaud,

H. Mestdagh

From the University

Hospital of Lille,

Lille, France
C. Maynou, MD, Professor of Orthopaedic Surgery X. Cassagnaud, MD, Orthopaedic Surgeon H. Mestdagh, MD Professor of Anatomy Department of Orthopaedic Surgery

University Hospital of Lille, 2 Avenue Oscar Lambret, 59000 Lille, France.

Correspondence should be sent to Professor C. Maynou, e-mail:

c-maynou@chru-lille.fr

(C)2005 British Editoria Society of Bone and Joint Surgery doi:10.1302/0301-620X.87B8. $14605 \$ 2.00$

$J$ Bone Joint Surg $[\mathrm{Br}]$ 2005;87-B:1096-101.

Received 6 May 2003;

Accepted after revision 10 November 2004

\title{
Function of subscapularis after surgical treatment for recurrent instability of the shoulder using a bone-block procedure
}

We compared the long-term function of subscapularis after the Latarjet procedure using
two surgical approaches. We treated 102 patients ( 106 shoulders) with a mean age of 26.8
years ( 15 to 51 ) with involuntary unidirectional recurrent instability. The operation was
carried out through an L-shaped incision with trans-section of the upper two-thirds of the
muscle in 69 cases and with a subscapularis split in 37 . All clinical results were assessed by
the Rowe and the Duplay scores and the function of subscapularis by evaluating the
distance and strength at the lift-off position. Bilateral CT was performed in 77 patients for
assessment of fatty degeneration. The mean follow-up was 7.5 years $(2$ to 15 ) and $18 \%$ of
cases were lost to follow-up. The mean Duplay score was 82 of 100 for the L-shaped
incision group and 90 of 100 for those with a subscapularis split ( $p=0.02$ ). The mean fatty
degeneration score was 1.18 after an L-shaped incision compared with 0.12 after
subscapularis split ( $p=0.001$ ). The subscapularis split approach is therefore recommended.

The use of the coracoid process to stabilise the shoulder was first described by Oudard ${ }^{1}$ in 1923. He introduced a bone graft into a split in the horizontal part of the coracoid apophysis to fill the coracoglenoidal space. Screwing of the coracoid process on to the anteroinferior side of the glenoid at the level of the anterior glenoid rim was described by Latarjet $^{2}$ in 1954. In his original technique, subscapularis was incised vertically along the whole of its length, then sutured with an overlap.

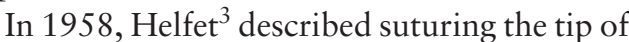
the coracoid process to the preglenoidal fibrous plane by splitting subscapularis. The aim was to create a hammock to stabilise the shoulder in maximal abduction and external rotation. Patte et $\mathrm{al}^{4}$ proposed changes in 1980 to Latarjet's technique by developing the concept of anterior triple locking, which involved a bone-block effect, suturing of the coracoacromial ligament to the medial capsular flap with preservation of the lower third of the tendon of subscapularis. Torg et $\mathrm{al}^{5}$ preserved the whole of subscapularis, which they flattened down whereas Weaver and Derkash ${ }^{6}$ only divided the lower part of the muscle.

Our aim was to analyse the function and CT appearance of subscapularis by comparing two different incision techniques; an L-shaped transsection and splitting of subscapularis. The capsule was always opened vertically and the bone graft positioned on to the anteroinferior rim of the glenoid.

\section{Patients and Methods}

In a preliminary study a control group of 60 individuals without symptoms in the shoulder girdle was assessed. There were 24 women and 36 men with a mean age of 31 years (23 to 53). Fifty were right-handed and ten were left-handed.

The lift-off test, described by Gerber and Krushell, ${ }^{7}$ was performed bilaterally, and the distance between the patient's hand and the dorsal plane was measured. The mean of two measurements performed three minutes apart was recorded.

The lift-off test was performed for five seconds in order to assess the power produced by subscapularis. The force produced during this manoeuvre was assessed using a dynamometer (Fig. 1). A second measurement was made after rest for three minutes and the mean of these two values was used. These measurements were performed bilaterally and compared using Student's $t$-test.

Between 1984 and 1998, 125 patients were operated on for instability of the shoulder using a bone-block procedure. Of these, 23 were lost to follow-up, leaving 102 (106 bone-block procedures) in the study. There were 85 men and 17 women with a mean age of 26.8 years ( 15 to 51) at the time of the operation. Of these, 77 (80 bone-block procedures) were examined 


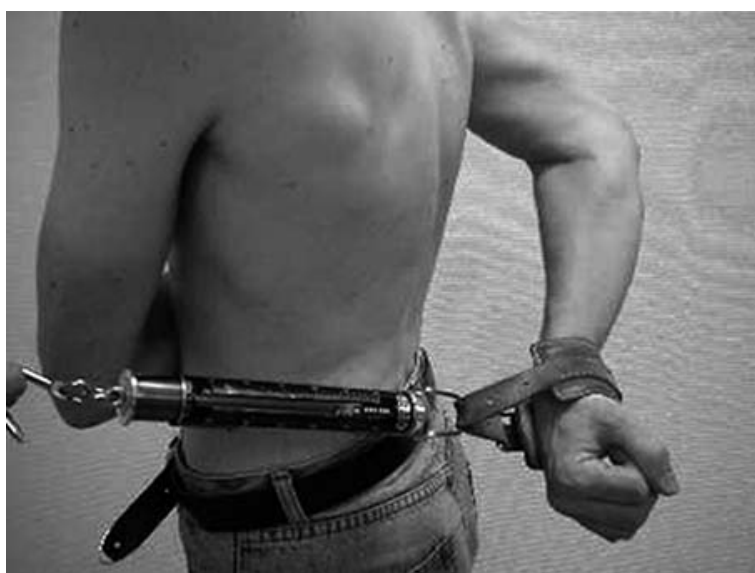

Fig. 1

Diagram showing the measurement of the strength of subscapularis.

clinically and with plain radiographs and CT scans, 25 (26 bone-block procedures) replied to a telephone questionnaire, and six had plain radiography only. Of the patients, $87(85 \%)$ practised sport $(46(45 \%)$ competitive sports and $41(40 \%)$ leisure sports) and $42(48 \%)$ of them an at-risk sport which involved blocked throwing movements. The dominant arm was operated on in 61 shoulders $(58 \%)$.

In the overall series of 106 shoulders, true recurrent dislocation occurred in 73 cases $(68.9 \%)$, recurrent subluxation in $24(22.6 \%)$ and both subluxation and dislocation in six $(5.6 \%)$. In all patients the instability was unidirectional and involuntary. Pre-operative radiological imaging showed $49(46.2 \%)$ of the lesions affected the anteroinferior border of the glenoid and $53(50.5 \%)$ the cephalic notch on the humeral head. Grade-I arthritis, according to the classification of Salmison and Prieto ${ }^{8}$ was evident in seven patients $(6.8 \%)$.

Pre-operative arthro-CT showed no tears of subscapularis. There were five cases of partial tear of the undersurface of the tendon of supraspinatus.

A deltopectoral approach was used. Subscapularis was opened using trans-section with an inverted ' $\mathrm{L}$ ' incision, preserving the lower third of the tendon, in 69 operations $(65 \%)$. In the remaining $37(35 \%)$, subscapularis and its tendon were split in the axis of the muscle fibres (Figs 2 and $3)$. The bone block was positioned at the level of the anterior rim of the glenoid and below its equator. Fixation was with one screw in 84 cases and two screws in 22 cases. The ' $\mathrm{L}$ ' incision in the tendon of subscapularis was repaired using non-reabsorbable woven sutures. When a split was performed, the edges were brought together using two simple reabsorbable sutures. Post-operatively, passive physiotherapy of the shoulder was undertaken for 15 days, followed by active exercises, excluding external rotation, for six weeks.

The mean follow-up was 7.5 years ( 2 to 15 ). In 82 cases $(77.3 \%)$ follow-up was more than five years. Outcome was

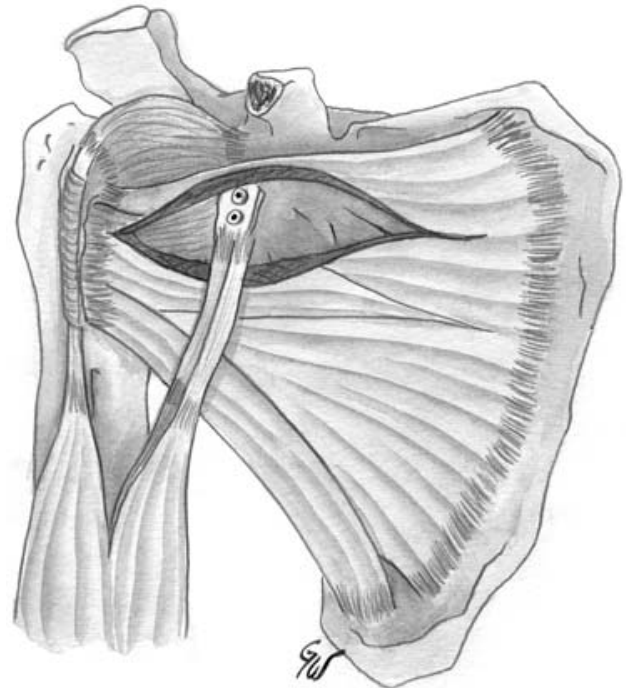

Fig. 2

Diagram showing splitting of subscapularis.

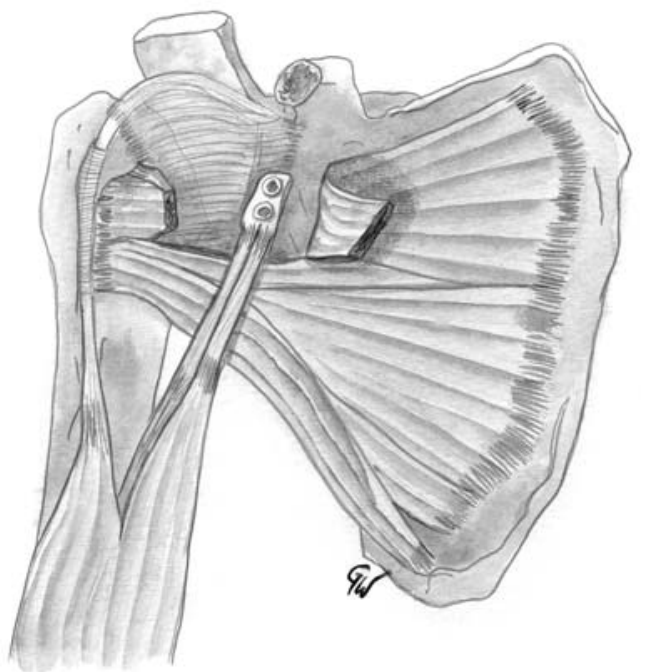

Fig. 3

Diagram showing the L-shaped incision of subscapularis.

assessed using scores described by Rowe, Patel and Southmayd ${ }^{9}$ and Walch (Duplay score).$^{10}$ Clinical assessment for signs of generalised laxity (Beighton score), signs of subacromial impingement or rotator cuff tear, and for residual anterior instability (apprehension in maximal abduction and external rotation, fulcrum test and blocked throwing) was also performed. Measurement of the distance from the hand to the back and of the muscle power from Gerber's lift-off test, as described for the control 


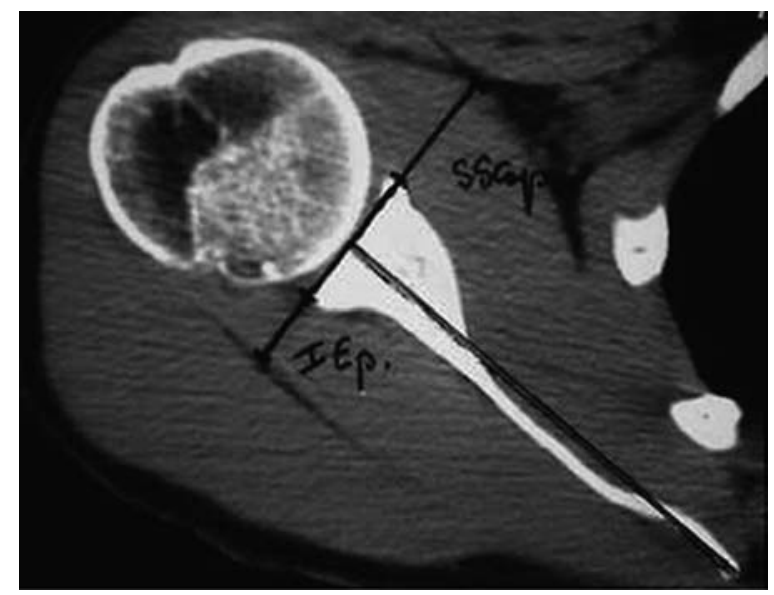

Fig. 4

CT showing the method of measurement of the relative atrophy of subscapularis.

group, was used to assess the function of subscapularis. Plain radiological examination included bilateral anteroposterior and neutral rotation position views, internal and external, a Lamy profile and a Bernageau glenoid profile.

Evidence of glenohumeral osteoarthritis on the anteroposterior or neutral rotation views, was classified according to the criteria of Samilson and Prieto. ${ }^{8}$ The position of the bone block, its changing appearance (lysis, pseudoarthrosis, migration), the length of the screws and their bicortical character, were estimated from the Lamy and Bernageau profiles.

CT assessment with thick window slices $(5 \mathrm{~mm})$ of the soft tissues was performed at the tip of the coracoid process of the healthy side and the operated side (inferred from the scout view of the healthy side). ${ }^{11}$

In order to measure atrophy of subscapularis, a tangent to the surface of the glenoid was drawn at the level of the tip of the coracoid apophysis, and the respective thicknesses of subscapularis and infraspinatus were measured on this straight line (Fig. 4). Individual variations in muscle volume and the differences depending on whether the patients were right or left-handed, were eliminated by taking into account only the ratio between the measurements of thickness made for these two muscles. The figures obtained therefore allowed us to compare the relative atrophy of subscapularis on the operated side with that on the healthy side. These measurements could not be compared with the pre-operative status because of absence of information on the preoperative arthro-CT scan.

Statistical analysis. We used the chi-squared and Student's $t$-tests for comparison between groups. A p value $<0.05$ was considered statistically significant.

\section{Results}

Control group. While Gerber's lift-off test was being performed, the mean hand-to-back distance was $9 \mathrm{~cm} \mathrm{(4} \mathrm{to} \mathrm{16)}$ on the dominant side (D) and $11 \mathrm{~cm}$ (4 to 18) on the nondominant side (d). The non-dominant side was therefore more supple with a $\mathrm{D} / \mathrm{d}$ ratio of $81.3 \%(\mathrm{p}<0.0001)$.

The mean muscle power produced to maintain the lift-off test manoeuvre against resistance was $8 \mathrm{~kg}$ ( 3 to 13) on the dominant and $7 \mathrm{~kg}$ ( 2 to 10 ) on the non-dominant side. The muscle power produced by the dominant side was therefore $1 \mathrm{~kg}$ more with a ratio $\mathrm{D} / \mathrm{d}$ of $124 \%(\mathrm{p}<0.0001)$. These ratios were the same for both men and women. The ratios calculated in this way for the control group were used to weight the results obtained in the operated patients with respect to arm dominance.

Operated group. Overall, the mean Duplay score was 84.8 of 100 (10 to 100) points with good and excellent results in $81(76.4 \%)$. The mean Rowe score was 89 of 100 with a satisfactory result in 93 patients $(88 \%)$. There was only one case of recurrent dislocation which occurred four years after the operation. Fourteen patients $(13.2 \%)$ complained of residual apprehension.

The mean mobility score according to the Duplay score ${ }^{10}$ was 19.3 of 25 points, with 67 patients $(63.2 \%)$ obtaining the maximum score. The mean external rotation of the elbow towards the body was $57.4^{\circ}\left(10^{\circ}\right.$ to $\left.90^{\circ}\right)$ on the operated side, compared with $66.9^{\circ}\left(30^{\circ}\right.$ to $\left.100^{\circ}\right)$ on the healthy side. The mean deficit of external rotation on the operated side was $\geq 30^{\circ}$ in nine shoulders $(8.5 \%)$. This mean deficit was smaller for the dominant $\left(8^{\circ} ;-20^{\circ}\right.$ to $\left.30^{\circ}\right)$ than for the non-dominant shoulders $\left(11.4^{\circ} ;-35^{\circ}\right.$ to $\left.15^{\circ}\right)(\mathrm{p}=0.003)$.

External rotation in $90^{\circ}$ of abduction showed a mean deficit of $10.6^{\circ}\left(10^{\circ}\right.$ to $\left.70^{\circ}\right)$ for the operated side compared with the healthy side. This mean deficit was smaller for the dominant $\left(8.1^{\circ} ;-70^{\circ}\right.$ to $\left.10^{\circ}\right)$ compared with the non-dominant shoulders $\left(14.1^{\circ} ;-35^{\circ}\right.$ to $\left.0^{\circ}\right)$.

The mean internal rotation with the hand behind the back on the operated side was 2.2 vertebral levels less than that on the healthy side. Position 2 and 3 rotation of the operated side was limited by $9.5^{\circ}$ and $7.6^{\circ}$ respectively, compared with the healthy side.

The mean hand-to-back distance in the lift-off position was $7.1 \mathrm{~cm}$ on the operated side and $9 \mathrm{~cm}$ on the healthy side $(\mathrm{p}<0.0001)$. There were no differences with respect to whether the dominant or the non-dominant arm was operated upon.

The mean muscle power from the lift-off test was $2 \mathrm{~kg}$ less on the operated side than on the healthy side $(\mathrm{p}=$ 0.0001) (Table I). The older the patients were at the time of the surgery the more the muscle power produced (mean 5.6 $\mathrm{kg}$; SD 2.40) was altered by the operation $(\mathrm{p}=0.0009)$. The loss of muscle power (mean $5.6 \mathrm{~kg}$; SD 2.4) was statistically linked to a worse Duplay score (mean 84.8 points; SD 20.39 ) and to a greater loss of internal rotation with the hand behind the back (mean -2.19 vertebral levels; SD 2.7) $(p=0.0001)$. After weighting, the loss of muscle power was greater for the operated dominant (weighted muscle power $=60.3 \%)$ than for the operated non-dominant arms (weighted muscle power $=85.5 \%$ ). The patients therefore 
Table I. Comparative results of the strength of subscapularis and the hand-to-back distance (mean; range) between the operated and healthy sides weighted or not by the values obtained in the control group

\begin{tabular}{lllll}
\hline & Operated side & Healthy side & $\begin{array}{l}\text { Unweighted } \\
\text { operated/healthy (\%) }\end{array}$ & $\begin{array}{l}\text { Weighted } \\
\text { operated/healthy (\%) }\end{array}$ \\
\hline Hand-to-back distance $(\mathrm{cm})$ & $7.1(0$ to 16$)$ & $9.3(5$ to 18$)$ & 76 & 75 \\
Subscapularis strength $(\mathrm{kg})$ & $5.6(0$ to 11$)$ & $7.6(3$ to 13$)$ & 74 & 71
\end{tabular}

Table II. Clinical results comparing the two surgical approaches

\begin{tabular}{llll}
\hline & L-shaped incision & Subscapularis & p value \\
\hline Mean age in yrs (range) & $27(15$ to 50$)$ & $26.2(17$ to 51$)$ & \\
Men:women (\%) & 18 & 11 & \\
Mean follow-up (mths) & $104(24$ to 180$)$ & $61.6(24$ to 120$)$ & \\
Number of shoulders (patients) & $69(65)$ & $37(35)$ & \\
Dominant arm involved (\%) & 68 & 44 & 0.02 \\
Mean Duplay score & $82 / 100$ & $90 / 100$ & 0.00 \\
Mean mobility score & $17.4 / 25$ & $22.9 / 25$ & $10^{-6}$ \\
Mean loss medial rotation & 3 vertebral levels & 0.6 vertebral levels & $10^{-6}$ \\
Mean hand-to-back distance (cm) & 5.6 & 9.3 & 0.0005 \\
Mean muscle strength (kg) & 4.8 & 6.7 & \\
\hline
\end{tabular}

recovered power in subscapularis better (mean $60.34 \%$; SD 26.6) when the non-dominant side (mean $85.1 \%$; SD 28 ) was operated on $(p=0.0001)$. Those with residual apprehension had a greater reduction in muscle power than those with a stable shoulder, with or without weighting. When residual apprehension was present, the mean weighted muscle power was $54.9 \%$ compared with $74.3 \%$ if the shoulder was stable $(\mathrm{p}=0.004)$.

Measurement of the hand-to-back distance (mean 7.1 cm; SD 3.7) and muscle power (mean $5.6 \mathrm{~kg}$; SD 2.4) from the lift-off test correlated strongly $(\mathrm{p}=0.0001)$.

Gender did not influence the clinical outcome. The two groups were not randomised and were not identical. The mean follow-up of the group with an L-shaped incision was 104 months (24 to 180 ) and of those with subscapularis split 61.6 months (24 to 120 ). Physiotherapy with external rotation was started early in the group with a subscapularis split (six weeks) compared with that with an L-shaped incision (eight weeks). More dominant arms were operated on using an inverted L-shaped incision $(68 \%)$ than by a subscapularis split (44\%) (Table II).

The mean total Duplay score out of 100 was 82.1 (25 to $100)$ for those with the L-shaped incision $(\mathrm{p}=0.02)$ and 89.9 points (65 to 100 ) for the group with a subscapularis split. External rotation in the plane of the scapula was more limited on the healthy side in patients with an L-shaped incision $(87.4 \%$ compared with $93.5 \% ; p=0.03)$. Loss of internal rotation with the hand behind the back reached a mean of three vertebral levels in the L-shaped group compared with 0.6 levels in the group with subscapularis split $(\mathrm{p}<$ 0.001 ). The mean hand-to-back distance measured $5.6 \mathrm{~cm}$ in the group with an L-shaped incision and $9.4 \mathrm{~cm}$ in those with subscapularis split $(\mathrm{p}<0.001)$. After weighting for the dominant or non-dominant arms, this distance was $65.2 \%$ of the theoretical value for the group with the L-shaped incision and $94.3 \%$ for those with subscapularis split.
The mean muscle power during the lift-off test was also greater after subscapularis split $(6.7 \mathrm{~kg})$ than after an Lshaped incision $(4.8 \mathrm{~kg})(\mathrm{p}=0.0005)$. After weighting the muscle power was $58.5 \%$ of its theoretical value for those with an L-shaped incision and $92.1 \%$ for those with a subscapularis split. The four patients in whom the distance and muscle power during the lift-off test of the operated arm were zero, had an L-shaped incision. At follow-up inferior laxity, assessed by means of a sulcus test, was more frequently observed after subscapularis split than after an L-shaped incision $(\mathrm{p}=0.01)$. The sulcus test was present in nine shoulders $(13 \%)$ with an L-shaped incision and in 15 $(40.5 \%)$ with subscapularis split.

\section{CT imaging}

Fatty degeneration of subscapularis. According to the classification of Goutallier et al, ${ }^{11}$ the mean fatty degeneration of subscapularis was 0.76 ( 0 to 4 ) on the operated side and 0.054 ( 0 to 2 ) on the healthy side ( $\mathrm{p}=0.001)$.

The mean age of patients without fatty degeneration was 25.8 years (SD 7.7) and with fatty degeneration was 32 years (SD 10.3). The older the patient was at the time of the operation, the greater was the fatty degeneration at followup $(\mathrm{p}=0.006)$. Fatty degeneration was observed in 15 $(31.9 \%)$ shoulders on the dominant side and in only three $(9 \%)$ shoulders on the non-dominant side. Fatty degeneration was greater for the dominant shoulders $(p=0.02)$, but the length of follow-up and physiotherapy had no statistical significance. The mean value for fatty degeneration was 1.18 (0 to 4 ) after an L-shaped incision and 0.12 (0 to 2) after subscapularis split $(\mathrm{p}=0.001)$. Only four patients were aged over 45 years at the time of surgery and fatty degeneration was therefore likely to be due to surgery rather than to age.

The mean total Duplay score was 71.7 when fatty degeneration was present and 87.1 when it was absent $(\mathrm{p}=0.006)$. 


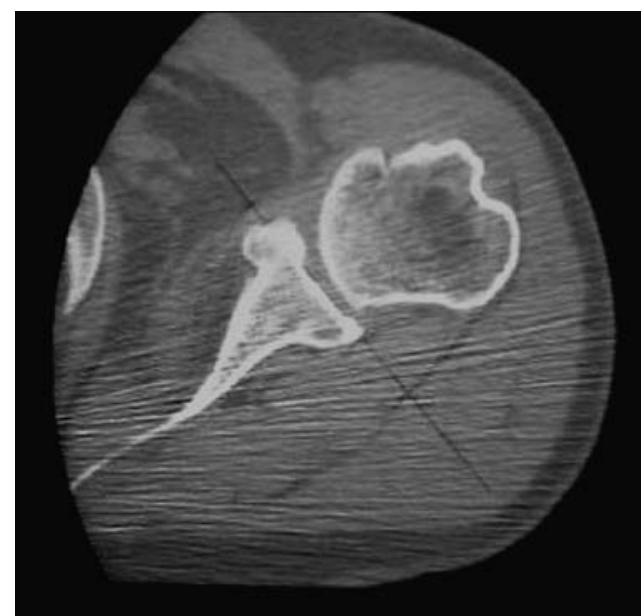

Fig. 5

CT showing fatty degeneration of subscapularis.

Six of the nine patients with poor results according to the Duplay score, had fatty degeneration of more than stage 2 . Fatty degeneration of stage 3 or greater was accompanied by persistent apprehension of $35.3 \%$ (Fig. 5).

Patients without fatty degeneration of the subscapularis had a mean hand-to-back distance of $7.9 \mathrm{~cm}$ (SD 3.3) and mean muscle power of $6.3 \mathrm{~kg}$ (SD 1.8); while patients with fatty degeneration had a mean hand-to-back distance of 4.3 $\mathrm{cm}$ (SD 3.7) and mean muscle power of $3.3 \mathrm{~kg}$ (SD 2.8). The hand-to-back distance and muscle power from the lift-off test were significantly altered when subscapularis was degenerative $(\mathrm{p}=0.0001)$. In addition, the loss of internal rotation with the hand behind the back reached a mean of five vertebral levels when degeneration was present and 1.3 levels if the muscle was healthy $(p=0.0001)$.

A study of fatty degeneration of infraspinatus showed no statistically significant difference between the operated (mean 0.45) and the healthy sides (mean 0.11). This was independent of the length of follow-up, the patient's age at the time of the operation and at follow-up, and dominance or non-dominance. However, it was linked to the length of the osteosynthesis screw used for the bone-block procedure when this extended beyond the posterior cortical bone of the scapula by more than $1 \mathrm{~cm}$ (14 cases) ( $\mathrm{p}=0.0001)$.

CT suggested that there were three cases in the L-shaped group of rupture of the upper third of subscapularis because of the absence of muscle tissue next to the medial side of the lesser tuberosity. In these three cases, the hand- to-back distance (mean $2.7 \mathrm{~cm}$; SD 2.5) and muscle power (mean $2 \mathrm{~kg}$; SD 2) from the lift-off test were considerably altered $(\mathrm{p}=0.02)$ and in two, fatty degeneration was advanced (stages 3 and 4).

Atrophy of subscapularis (Table III). The relative thickness of subscapularis was statistically less on the operated side (mean $0.9 \mathrm{~cm}$; SD 0.37 ) than on the healthy side (mean 1.16; SD 0.24) ( $\mathrm{p}=0.0001)$ and was more than $0.5 \mathrm{~cm}$ in 25 cases.

Atrophy of subscapularis correlated with the patient's age $(26.8$ years $)$ at the time of the operation $(p=0.009)$ and whether the dominant arm was operated on or not $(\mathrm{p}=0.001)$. The atrophy was closely related to the method used for opening subscapularis ( $\mathrm{p}<0.001)$, with a mean ratio of 0.71 for L-shaped incision and 1.2 for subscapularis split. Therefore, after the L-shaped, a relative loss in the thickness of 0.44 occurred, compared with the healthy side, whereas there was no loss with subscapularis split.

This atrophy affected the clinical outcome (Duplay score; $\mathrm{p}=0.005)$ and the amplitude of internal rotation with the hand behind the back which showed a mean loss of four vertebral levels $(\mathrm{p}=0.0001)$.

The hand-to-back distance and muscle power produced on the lift-off manoeuvre were significantly affected by muscle atrophy $(\mathrm{p}=0.0001)$.

Fatty degeneration and muscle atrophy were closely linked $(\mathrm{p}=0.0001)$ since in the 25 cases in which the relative loss of thickness compared with the healthy side was greater than 0.5 , degeneration of more than stage 2 was observed in 16 .

\section{Discussion}

Our aim was to assess the function and fatty degeneration of subscapularis, after stabilisation of the shoulder by a bone-block procedure, particularly focusing on the longterm consequences of the method used for opening subscapularis. With a clinical, radiological and CT follow-up for a mean of 7.5 years, our study provided one of the longest periods of follow-up for pre-glenoid bone-block procedures. To our knowledge, only Singer, Kirkland and Emery $^{12}$ (mean 20.5 years), Allain, Goutallier and Glorion $^{13}$ (mean 14.3 years), Banas et al $^{14}$ (mean 8.6 years) and Benammar et $\mathrm{al}^{15}$ (mean 8 years) had a longer followup period.

Our preliminary study in individuals without any scapular pathology showed significant differences in mobility and muscle power, depending on whether the patients were leftor right-handed. The dominant arm was less supple but

Table III. Measurement of muscle atrophy of subscapularis (cm)

\begin{tabular}{llll}
\hline & Operated side & Healthy side & Loss of thickness \\
\hline Overall series (mean; range) & $0.91(0$ to 1.7) & 1.17 (0.52 to 1.75) & 0.26 \\
L-shaped incision & 0.71 & 1.5 & 0.44 \\
Subscapularis split & 1.2 & 1.2 & 0.0 \\
\hline
\end{tabular}


stronger than the non-dominant arm. We, therefore, took this into account in our assessment of clinical outcome.

Our clinical and CT results show the superiority of subscapularis split along the muscle fibres over the classical inverted L-shaped incision. The latter appeared to cause greater loss of range and power of internal rotation, as assessed by Gerber's lift-off test, and more restriction of external rotation than did splitting the tendon. The overall function of the shoulder, assessed by the Rowe and Duplay scores, was also affected adversely. Scarring fibrosis and the inevitable shortening of subscapularis after trans-section, are probably responsible for the differences between the two methods. Our study confirmed the results of Picard et $\mathrm{al}^{16}$ who observed a significant loss of amplitude and muscle power after complete vertical sectioning of subscapularis.

In our series the clinical results correlated with the CT findings, which showed that loss of internal and external rotation and the loss of muscle power correlated with more marked fatty degeneration and muscle atrophy and was more noticeable after the L-shaped incision.

The clinical consequences of fatty degeneration are important since six of the nine poor clinical results, showed fatty degeneration of stage 2 or more. Other factors were also associated with fatty degeneration and included the age of the patient at surgery. This phenomenon is already known in rotator-cuff disease.

Muscle atrophy was more marked if the operation was performed on the dominant arm, but the L-shaped incision was performed on $68 \%$ of these, which may explain the difference. The surgical approach appeared to be the main cause of the muscle atrophy. There were no differences between the two groups as to age, gender or loss to followup.

In three cases, we suspected that there was a tear of subscapularis as shown on the CT scans by the absence of muscle tissue anterior to the lesser tuberosity. This was probably due to the sutures becoming loose.

Inferior subluxation of the shoulder was more frequently observed after subscapularis split than after the L-shaped incision but had no clinical consequences. When the coracoid transplant was taken, sectioning of the coracohumoral ligament inevitably produced opening of the rotator interval. When the subscapularis splits were performed, this space was not repaired. The L-shaped incision, however, was repaired in the upper part of subscapularis thus partially closing the rotator interval, and may explain why less downward movement of the humerus was observed. We recommend closure of the rotator interval to avoid inferior laxity in the subscapularis split approach. In the light of these results, it appears to be preferable to approach the anterior part of the glenoid using a simple split of subscapularis. The anatomical and clinical complications are fewer than those after the inverted L-shaped incision. In our series, subscapularis split did not influence the positioning of the bone block and did not cause any specific morbidity.

No benefits in any form have been received or will be received from a commercial party related directly or indirectly to the subject of this article.

\section{References}

1. Oudard M. La luxation récidivante de l'épaule, procédé opératoire. J Chir 1923;23: 13-25 (in French).

2. Latarjet M. Treatment of recurrent dislocation of the shoulder. Lyon Chir 1954;49: 994-1003.

3. Helfet AJ. Coracoid transplantation for recurring dislocation of the shoulder. J Bone Joint Surg [Br] 1958;40-B:198-202.

4. Patte D, Bernageau J, Rodineau J, et al. Unstable painful shoulders. Rev Chir Orthop Reparatrice Appar Mot 1980;66:157-65 (in French).

5. Torg JS, Balduini FC, Bonci C, et al. A modified Bristow-Helfet-May procedure for recurrent dislocation and subluxation of the shoulder: report of two hundred and twelve cases. J Bone Joint Surg [Am] 1987;69-A:904-13.

6. Weaver JK, Derkash S. Don't forget the Bristow-Latarjet procedure. Clin Orthop 1994;308:102-10.

7. Gerber CH, Krushell RJ. Isolated rupture of the tendon of the subscapularis muscle: clinical features in 16 cases. J Bone Joint Surg [Br] 1991;73-B:389-94.

8. Samilson RL, Prieto V. Dislocation arthropathy of the shoulder. J Bone Joint Surg [Am] 1983;65-A:456-60.

9. Rowe CR, Patel D, Southmayd WW. The Bankart procedure: a long-term endresult study. J Bone Joint Surg [Am] 1978;60-A:1-16.

10. Walch G. Luxation récidivante antérieure de l'épaule. Rev Chir Orthop 1991;77 (Suppl 1):177-91 (in French)

11. Goutallier D, Postel JM, Bernageau J, Lavau L, Voisin MC. Fatty muscle degeneration in cuff ruptures: pre- and postoperative evaluation by CT scan. Clin Orthop 1994;304:78-83

12. Singer GC, Kirkland PM, Emery RJH. Coracoid transposition for recurrent anterior instability of the shoulder: a 20-year follow-up study. J Bone Joint Surg [Br] 1995; 77-B:73-6.

13. Allain J, Goutallier D, Glorion C. Long term results of the Latarjet procedure for the treatment of anterior instability of the shoulder. J Bone Joint Surg [Am] 1998, 80-A:841-52.

14. Banas MP, Dalldorf PG, Sebastianelli WJ, De Haven KE. Long-term followup of the modified Bristow procedure. Am J Sports Med 1993;21:666-71.

15. Benammar MN, Saragaglia D, Legrand JJ, Faure C, Butel J. Latarjet's surgery in recurrent anterior dislocations of the shoulder: 117 cases with an 8-year follow-up. Rev Chir Orthop Reparatrice Appar Mot 1986;72:447-54 (in French).

16. Picard F, Saragaglia D, Montbarbon E, et al. Anatomo-clinical consequences of the vertical sectioning of the subscapular muscle in Latarjet intervention. Rev Chir Orthop Reparatrice Appar Mot 1998;84:217-23 (in French). 